Accepted for publication in The Astrophysical Journal

Preprint typeset using $\mathrm{LAT}_{\mathrm{E}} \mathrm{X}$ style emulateapj v. 08/22/09

\title{
THE SLOAN LENS ACS SURVEY. VII. ELLIPTICAL GALAXY SCALING LAWS FROM DIRECT OBSERVATIONAL MASS MEASUREMENTS ${ }^{1}$
}

\author{
Adam S. Bolton ${ }^{2,3}$, Tommaso Treu ${ }^{4,5}$, Léon V. E. Koopmans ${ }^{6}$, Raphä̈l Gavazzi ${ }^{4,7}$, Leonidas A. Moustakas ${ }^{8}$, \\ Scott Burles ${ }^{9}$, David J. Schlegel ${ }^{10}$, And Randall Wayth ${ }^{3}$ \\ Accepted for publication in The Astrophysical Journal
}

\begin{abstract}
We use a sample of 53 massive early-type strong gravitational lens galaxies with well-measured redshifts (ranging from $z=0.06$ to 0.36 ) and stellar velocity dispersions (between 175 and $400 \mathrm{~km} \mathrm{~s}^{-1}$ ) from the Sloan Lens ACS (SLACS) Survey to derive numerous empirical scaling relations. The ratio between central stellar velocity dispersion and isothermal lens-model velocity dispersion is nearly unity within errors. The SLACS lenses define a fundamental plane (FP) that is consistent with the FP of the general population of early-type galaxies. We measure the relationship between strong-lensing mass $M_{\text {lens }}$ within one-half effective radius $\left(R_{e} / 2\right)$ and the dimensional mass variable $M_{\text {dim }} \equiv G^{-1} \sigma_{e 2}^{2}\left(R_{e} / 2\right)$ to be $\log _{10}\left[M_{\text {lens }} / 10^{11} M_{\odot}\right]=(1.03 \pm 0.04) \log _{10}\left[M_{\operatorname{dim}} / 10^{11} M_{\odot}\right]+(0.54 \pm 0.02)$ (where $\sigma_{e 2}$ is the projected stellar velocity dispersion within $\left.R_{e} / 2\right)$. The near-unity slope indicates that the massdynamical structure of massive elliptical galaxies is independent of mass, and that the "tilt" of the SLACS FP is due entirely to variation in total (luminous plus dark) mass-to-light ratio with mass. Our results imply that dynamical masses serve as a good proxies for true masses in massive elliptical galaxies. Regarding the SLACS lenses as a homologous population, we find that the average enclosed $2 \mathrm{D}$ mass profile goes as $\log _{10}\left[M(<R) / M_{\mathrm{dim}}\right]=(1.10 \pm 0.09) \log _{10}\left[R / R_{e}\right]+(0.85 \pm 0.03)$, consistent with an isothermal (flat rotation curve) model when de-projected into 3D. This measurement is inconsistent with the slope of the average projected aperture luminosity profile at a confidence level greater than $99.9 \%$, implying a minimum dark-matter fraction of $f_{\mathrm{DM}}=0.38 \pm 0.07$ within one effective radius. We also present an analysis of the angular mass structure of the lens galaxies, which further supports the need for dark matter inside one effective radius.

Subject headings: gravitational lensing — galaxies: elliptical — surveys
\end{abstract}

\section{INTRODUCTION}

Elliptical galaxies are simple in appearance, but their internal structure is resistant to elementary Newtonian deduction because the primary luminous tracers of their gravity - stars - move not on "cold" circular orbits but on "hot" randomized orbits with a broad distribution in phase space (e.g., Bertola \& Capaccioli 1975;

\footnotetext{
${ }^{1}$ Based on observations made with the NASA/ESA Hubble Space Telescope, obtained at the Space Telescope Science Institute, which is operated by AURA, Inc., under NASA contract NAS 526555. These observations are associated with programs \#10174, \#10494, \#10587, \#10798, and \#10886.

2 Beatrice Watson Parrent Fellow, Institute for Astronomy, University of Hawai'i, 2680 Woodlawn Dr., Honolulu, HI 96822, USA (bolton@ifa.hawaii.edu)

${ }^{3}$ Harvard-Smithsonian Center for Astrophysics, 60 Garden St. Cambridge, MA 02138, USA (rwayth@cfa.harvard.edu)

${ }^{4}$ Department of Physics, University of California, Santa Barbara, CA 93101, USA (tt@physics.ucsb.edu)

${ }^{5}$ Sloan Fellow, Packard Fellow

${ }^{6}$ Kapteyn Astronomical Institute, University of Groningen, P.O. Box 800, 9700AV Groningen, The Netherlands (koopmans@astro.rug.nl)

${ }^{7}$ Institut d'Astrophysique de Paris, UMR7095 CNRS \& Univ. Pierre et Marie Curie, 98bis Bvd Arago, F-75014 Paris, France (gavazzi@iap.fr)

8 Jet Propulsion Laboratory, California Institute of Technology, 4800 Oak Grove Drive, M/S 169-327, Pasadena, CA 91109, USA (leonidas@jpl.nasa.gov)

${ }^{9}$ Department of Physics and Kavli Institute for Astrophysics and Space Research, Massachusetts Institute of Technology, 77 Massachusetts Avenue, Cambridge, MA 02139, USA (burles@mit.edu)

${ }^{10}$ Physics Division, Lawrence Berkeley National Laboratory, Berkeley, CA 94720-8160, USA (djschlegel@lbl.gov)
}

Binnev 1976, 1978; Illingworth 1977). With detailed spatially resolved observations of the absorptionline kinematics of nearby elliptical galaxies, this difficulty can be attacked head-on through the use of dynamical modeling of the distribution function of stars in phase space (e.g., van der Marel 1991; Rix \& White 1992; Bertin. Saglia, \& Stiavelli 1992: Saglia. Bertin. \& Stiavelli 1992; Saglia et al. 1993; van der Marel \& Franx 1993; Merritt \& Saha 1993; Kuiiken \& Merrifield 1993; Gerhard 1993; Bender et al. 1994; Bertin et al. 1994; Rix et al. 1997; Kronawitter et al. 2000; Romanowsky \& Kochanek 2001; Saglia et al. 2000; Kronawitter et al. 2000; Gerhard et al. 2001 Cappellari et al. 2006). Such efforts require a very detailed level of analysis, and rely on assumptions of dynamical relaxation and the choice of correct dynamical model families.

Strong gravitational lensing offers an attractive complement to dynamical modeling, since it measures the total mass within the "Einstein radius" defined by the lensed images - a scale that is generally comparable to the effective radius of the luminous matter distribution in the case of galaxy-scale lenses. These lensing mass measurements are extremely robust, with few underlying assumptions and minimal model dependence (e.g., Kochanek 1991). The main limitation to strong lensing as a probe of elliptical galaxy structure has typically been the lack of sufficiently large and uniform lens samples. This limitation has been overcome in recent years by the Sloan Lens ACS Survey (SLACS: Bolton et al.|2006; Treu et al. 2006; Koopmans et al. 2006; Gavazzi et al. 
2007; Bolton et al. 2008; Gavazzi et al. 2008; hereafter Papers I-VI) and other surveys (e.g., Winn et al. 2000, 2001, 2002a.b; Myers et al. 2003; Browne et al. 2003; Maoz et al. 1993; Morgan et al. 2003; Wisotzki et al. 1993, 1996, 1999; Gregg et al.|2000; Wisotzki et al. 2002. 2004; Blackburne et al. 2008; Willis et al. 2005, 2006; Oguri et al. 2006; Inada et al. 2007; Oguri et al. 2007; Ratnatunga et al. 1999; Moustakas et al. 2007; Cabanac et al. 2007; Belokurov et al. 2007; Kubo \& Dell'Antonio 2008; Faure et al. 2008), which have begun to deliver statistically significant samples of strong lens galaxies by using systematic search techniques.

Previous studies have taken diverse approaches to the use of strong-lensing information for the measurement of early-type galaxy mass-density structure. Strong-lensing aperture masses have been used by Rusin. Kochanek. \& Keeton (2003) and Rusin \& Kochanek (2005) to provide ensemble constraints upon the mass-density structure of early-type galaxies. Lensing mass constraints have been combined in self-consistent fashion with stellar dynamics to constrain the mass structure of lenses on a system-by-system basis by the Lenses Structure and Dynamics Survey (LSD: Koopmans \& Treu 2002, 2003; Treu \& Koopmans 2002, 2003, 2004) and the SLACS Survey (Paper III). Kochanek (2002) and Kochanek et al. (2006) have used the time delays between multiple lensed quasar images, together with a given value for the Hubble parameter $H_{0}$ to measure the local mass slope of the lensing galaxies. Constraints on the mass profile of lenses based upon resolved lensed features have been published by Cohn et al. (2001), Muñoz et al. (2001), Wavth et al. (2005), Dve \& Warren (2005), Brewer \& Lewis (2006), and Dye et al. (2007). Bolton et al. (2007, hereafter B07) have used lensing aperture masses in combination with central stellar velocity dispersions and measured effective radii to demonstrate on an empirical scaling basis that the mass-density structure of massive elliptical lenses from the SLACS Survey is on average independent of mass, a result which we establish and explore in more detail in the current work.

In this work, we employ the mass, light, and velocity measurements of the full Hubble Space Telescope (HST) Advanced Camera for Surveys (ACS) gravitational lens sample from the SLACS Survey, published in Paper V, to derive numerous empirical scaling relations. The analysis of the initial SLACS sample in Paper III allowed us to measure an on-average isothermal massdensity structure; the current lens sample is large enough to investigate trends in structure with mass and velocity dispersion. We frame much of our analysis and discussion in terms of the Fundamental Plane scaling relation (FP; e.g., Diorgovski \& Davis 1987; Dressler et al. 1987; Bender et al.|1992; Renzini \& Ciotti 1993; Guzmán et al. 1993; Pahre et al. 1995, 1998b. a; Jørgensen et al. 1996; van Dokkum \& Franx 1996; Ciotti et al.|1996; Kelson et al.|1997; Graham \& Colless 1997; Scodeggio et al. 1998; Kochanek et al. 2000; Treu et al. 2001, 2002; Bertin et al. 2002: van Dokkum \& Stanford 2003; $\quad$ Bernardi et al. 2003; van de Ven et al. 2003; Truiillo et al. 2004; van der Wel et al. 2005; Paper II; B07) and the relationships between luminosity, "dynamical mass", and strong-lensing aperture mass that illuminate the structural explanation for the "tilt" of the FP. In the SLACS sample, we have a large number of early-type galaxies distributed across the higher-mass end of the FP, with uniform and high-quality measurements of the observables of redshift, surface brightness, velocity dispersion, and effective radius. In addition to these quantities, we have a full set of aperture masses-measurements of the total mass within the Einstein ring radius. These additional mass measurements add another dimension of physical constraint to the SLACS sample that is not available for other FP galaxy samples, thus offering the opportunity to break some of the degeneracy inherent in the physical interpretation of FP analyses.

This paper is arranged as follows. Section 2 gives an overview of our measurements and analysis techniques. Sections 36 present the various scaling relations defined by the SLACS sample, including the relative alignment and flattening of projected mass and light distributions (93), the relation between stellar and lensing velocitydispersion measurements (4), the FP and "mass plane" relations (\$5), and various perspectives on the relationship between dynamical mass, lensing mass, and luminosity ( 96 . In $\$ 7$ we make a robust determination of the ensemble average radial enclosed-mass profile of the SLACS sample. Section 8 provides an itemized summary of our results, and 99 provides a concluding discussion.

All computations in this work assume a general relativistic Friedmann-Robertson-Walker cosmology with matter-density parameter $\Omega_{\mathrm{M}}=0.3$, vacuum energydensity parameter $\Omega_{\Lambda}=0.7$, and Hubble parameter $H_{0}=70 \mathrm{~km} \mathrm{~s}^{-1} \mathrm{Mpc}^{-1}$.

\section{OVERVIEW OF SAMPLE, MEASUREMENTS, AND METHODS}

We consider the sample of 63 "grade-A" strong gravitational lenses presented in Paper V. For analyses employing stellar velocity dispersions, we restrict the sample to the 53 early-type lens systems with a median SDSS spectral signal-to-noise ratio of 10 or greater per $69 \mathrm{~km} \mathrm{~s}^{-1}$ pixel over the rest-frame range of 4100 to $6800 \AA$. The measurements upon which the current work is based are all presented in Paper V, and are described only briefly here.

We use stellar velocity dispersions measured from Sloan Digital Sky Survey (SDSS: York et al. 2000; Adelman-McCarthy et al. 2007) spectroscopy, which samples a $3^{\prime \prime}$-diameter circular fiber aperture centered on the target galaxy. We correct these measured velocity dispersions (which we denote by $\sigma_{\text {SDSS }}$ ) using the empirical power-law relation of Jørgensen et al. (1995) to uniform physical apertures of either $R_{e} / 8$ (to give a "central" velocity dispersion $\sigma_{e 8}$ ) or $R_{e} / 2\left(\sigma_{e 2}\right.$, for a spatial aperture more closely matched to that of the spectroscopy and of the strong-lensing features). We note that these corrections are quite small-RMS of $2.5 \%$ for $R_{e} / 2$ - and that the coefficients of logarithmic scaling relations we derive are not sensitive to the particular choice of velocity-normalization aperture. We use magnitudes, effective radii, projected axis ratios, and position angles for the lens galaxies measured from fitting de Vaucouleurs models to ACS-WFC F814W imaging. To convert from observed magnitudes to restframe luminosities, we first correct for Galactic dust ex- 
TABLE 1

ADOPTED ERRORS IN MEASURED DATA VALUES

\begin{tabular}{lc}
\hline \hline Measured parameter & Adopted error \\
\hline $\log _{10}$ Luminosity $L_{V}$ & $0.010 \mathrm{dex}$ \\
$\log _{10}$ Effective radius $R_{e}$ & $0.015 \mathrm{dex}$ \\
$\log _{10}$ Aperture mass $M$ & $0.010 \mathrm{dex}$ \\
$\log _{10}$ Surface brightness $I_{e}=L_{V} /\left(2 \pi R_{e}^{2}\right)$ & $0.020 \mathrm{dex}$ \\
$\log _{10}$ Velocity dispersion $\sigma$ & $0.030 \mathrm{dex}$ \\
\hline
\end{tabular}

tinction using the maps of Schlegel. Finkbeiner, \& Davis (1998), and apply $k$-corrections and evolutionary corrections. Our strong gravitational-lens models provide for each lens a robust measurement of the mass $M_{\text {Ein }}$ enclosed within the physical Einstein radius $R_{\text {Ein }}$ (derived from the angular Einstein radius $b$ together with redshifts and cosmology), for both singular isothermal ellipsoid (SIE: Kassiola \& Kovner 1993; Kormann et al. 1994; Keeton \& Kochanek 1998) and light-traces-mass (LTM) models for the lens-galaxy mass-density profile. For much of our analysis, we evaluate the strong-lensing aperture masses for a uniform physical aperture of $R_{e} / 2$ (one-half the effective radius of the luminous component), chosen to be fairly closely matched to the sample median Einstein radius $R_{\text {Ein }}$ of approximately $0.6 R_{e}$. The evaluation of the aperture mass at a radius other than $R_{\text {Ein }}$ introduces some dependence upon the assumed lens-mass model; we gauge this effect by using aperture masses from both SIE and LTM models, by checking for systematic residual correlations with the ratio $R_{\mathrm{Ein}} / R_{e}$ (of which we find none), and by verifying that our results do not change when derived using only the half of the sample with the smallest fractional aperture-mass difference between $R_{\text {Ein }}$ and $R_{e} / 2$. For an overview of the theory, phenomenology, and scientific applications of strong gravitational lensing, we refer the reader to Part 2 of Schneider, Kochanek, \& Wambsganss $(2006)$.

Unless otherwise noted, all scaling relations are fitted as linear and plane relationships in logarithmic space. We define the best fit as that which minimizes the total squared orthogonal distance from the line or plane to the set of sample data points. Before carrying out the fits, we scale all logarithmic data coordinates by their typical logarithmic errors, so as to apply a roughly uniform error metric across the multiple observables that span the spaces under consideration. The adopted errors are taken from the comparisons among multiple measurement techniques in Paper V, and are given in Table 1 We estimate errors on our fitted parameters using bootstrap re-sampling of the analysis sample (Efron 1979). Our reported errors are the square-root diagonal entries of the parameter covariance matrices constructed from the sets of bootstrap parameter estimates.

\section{THE COMPARATIVE ANGULAR STRUCTURE OF MASS AND LIGHT}

In addition to the measurement of aperture masses, the simplest strong-lens models also give a determination of the angular structure of the lens mass profile, in the form of either a mass axis ratio (for the SIE models) or an external shear magnitude (for the LTM models), along with the associated position angle. The implications of these measurements were discussed at length in Paper III; we briefly revisit the analysis here for the larger sample of lenses presented in Paper V.

First we examine the position-angle alignment between the major axes of the light profile (as determined by the de Vaucouleurs fit) and the mass profile (as determined from the SIE model). For the 58 lenses with light-profile axis ratios $q_{\text {stars }}<0.95$, the mean position-angle difference between mass and light profiles is $2.5^{\circ} \pm 2.4^{\circ}$. The RMS alignment is $18^{\circ}$, as compared with an intrinsic error of $7.5^{\circ}$ in the mass position angles from the analysis of Paper V. We can further restrict our attention to a subset of 29 of these lenses whose lensed images extend over a significant azimuth about the lens-galaxy center, for which the mass PA measurement is more tightly constrained, taken from the 32 lenses of the "ring subset" described in Paper V. In this case, we find a mean masslight PA difference of $1.4^{\circ} \pm 1.9^{\circ}$. From Paper V, the RMS error in the mass position angle measurement for the ring subset is approximately $2^{\circ}$. The RMS positionangle difference for the ring subset of lenses is $10^{\circ}$, equal to the value found in Paper III for the original SLACS sample.

Interestingly, the LTM plus external shear models of Paper V show a significant amount of preferential alignment of the "external" shear with the major-axis PA of the de Vaucouleurs surface-brightness model. Of the above-mentioned 29 well-constrained ("ring subset") lenses with $q_{\text {stars }}<0.95,17(59 \%)$ have their shear PA aligned to within $\pm 15^{\circ}$ of their luminosity PA, an angular range that only encompasses $17 \%$ of the meaningful range of variation $\left( \pm 90^{\circ}\right)$. If the shear were external and uncorrelated with the PA of the luminous distribution, this level of random alignment would have a probability of less than one in $10^{6}$. This result suggests that much of the shear required by the LTM lens models is not of environmental origin, but is instead compensation for an intrinsic shortcoming of the LTM lens models. The fact that the shear is aligned with the light position angles rather than being anti-aligned ( $90^{\circ}$ out of phase) shows that the lensed features require a stronger gravitational quadrupole at the locations of the lensed images than can be provided by the pure LTM models. This translates into a requirement either that the radial mass-density profile be less centrally concentrated than the light profile, or that the projected mass profile be flatter than the projected luminosity profile. In either case, the improbable alignment of "external shear" with the major axes of LTM lens models provides evidence against the lighttraces-mass hypothesis and thus in favor of some form of dark-matter component.

We also examine the relative flattening of mass and light as measured from SIE and de Vaucouleurs models. For the 57 grade-A lenses with early-type morphology, we find $\left\langle q_{\mathrm{SIE}} / q_{\mathrm{stars}}\right\rangle=1.02 \pm 0.02$ with an RMS deviation of 0.12 about the mean: i.e., mass and light have essentially the same projected axis ratio. As in Paper III, we see a trend from mass being rounder than light at lower masses towards mass being flatter than light at higher masses. Considering the subset of grade-A lenses with early-type morphology that both (1) are in the angularly well-constrained "ring subset", and (2) have mass and light position angles aligned to within $15^{\circ}$, this trend can be seen as a linear correlation coefficient of $q_{\mathrm{SIE}} / q_{\mathrm{stars}}$ with $\sigma_{\mathrm{SIE}}$ of $r=-0.665$, corresponding to a significance 
of $99.95 \%$.

\section{VELOCITY DISPERSIONS: STELLAR AND LENSING}

For SIE lens models, we may translate the measured Einstein radii $b$ into lens-model velocity dispersions $\sigma_{\mathrm{SIE}}$ through the relation

$$
b_{\mathrm{SIE}}=4 \pi \frac{\sigma_{\mathrm{SIE}}^{2}}{c^{2}} \frac{D_{\mathrm{LS}}}{D_{\mathrm{S}}},
$$

where $D_{\mathrm{LS}}$ and $D_{\mathrm{S}}$ are "angular-diameter distances" from lens (foreground galaxy) to source (background galaxy) and observer to source respectively. Though the conversion is strictly appropriate only for an isotropic, spherically symmetric, single-component density distribution with $\rho \propto r^{-2}$ (in 3D), it provides a robust velocity-scale measurement with extremely small statistical error, to be compared with the (usually noisier) central velocity dispersion $\sigma_{\text {stars }}$ of the stars within the lens galaxy. By considering bulge-plus-isothermal-halo models, Kochanek (1994) suggested that an approximate equality should hold between the central stellar line-ofsight velocity dispersion and the velocity-dispersion parameter of the isothermal halo. This equality has generally been confirmed in successively larger samples of lens galaxies with measured stellar velocity dispersions (Kochanek et al. 2000, LSD, Papers II and III), and the current SLACS lens sample allows the most significant determination to date.

Weighting with the statistical errors of the SDSS velocity dispersions (limited to a minimum error-bar size of $\left.\delta \sigma_{\text {stars }}=0.05 \sigma_{\text {stars }}\right)$, we find $\sigma_{\mathrm{SDSS}}=(0.948 \pm 0.008) \sigma_{\mathrm{SIE}}$ and $\sigma_{e 8}=(1.019 \pm 0.008) \sigma_{\mathrm{SIE}}$. Thus we see that the scaling $\sigma_{e 8}=\sigma_{\mathrm{SIE}}$ holds to within less than $3 \%$, and is essentially consistent with being exactly true on average at the current level of evidence. The RMS scatter about the relation is approximately 0.09 for $\sigma_{\text {SDSS }}$ and 0.1 for $\sigma_{e 8}$, somewhat larger than seen in Paper II. The reduced $\chi^{2}$ of this scatter when weighted by the errors as in the averaging is 2.7, showing evidence for intrinsic RMS scatter at the level of about $20 \mathrm{~km} \mathrm{~s}^{-1}$, or alternatively at the level of $7.5 \% \sigma_{\mathrm{SIE}}$ (i.e., the necessary value added in quadrature to the velocity errors to give a reduced $\chi^{2}$ of approximately unity). Figure 11 shows the ratio $f \equiv \sigma_{\text {stars }} / \sigma_{\text {SIE }}$ as a function of $\sigma_{\mathrm{SIE}}$. Note that our definition of $f$ follows the convention of Paper II, but is the inverse of the definition used in Kochanek et al. (2000).

The residuals about the mean $\sigma_{\text {stars }}-$ versus- $\sigma_{\text {SIE }}$ value are not significantly correlated with $\sigma_{\mathrm{SIE}}$, mass, luminosity, mass-to-light ratio, effective radius, or ratio of Einstein radius to effective radius. There is an apparent correlation with $\sigma_{\text {stars }}$, but this is an artifact of the relatively large statistical errors in that quantity. The lack of a correlation between $f$ and lens mass in particular indicates that the mass-dynamical structure of massive early-type galaxies is independent of mass. Likewise, the lack of correlation between $f$ and the ratio of Einstein radius $R_{\text {Ein }}$ to effective radius $R_{e}$ indicates that the universal density structure is nearly isothermal independently of dynamical modeling. These two results will be derived quantitatively in 6 and 97 below, and discussed further in 99 .

Auger (2007) has reported a significant correlation for the 15 SLACS lenses analyzed in Paper III between the

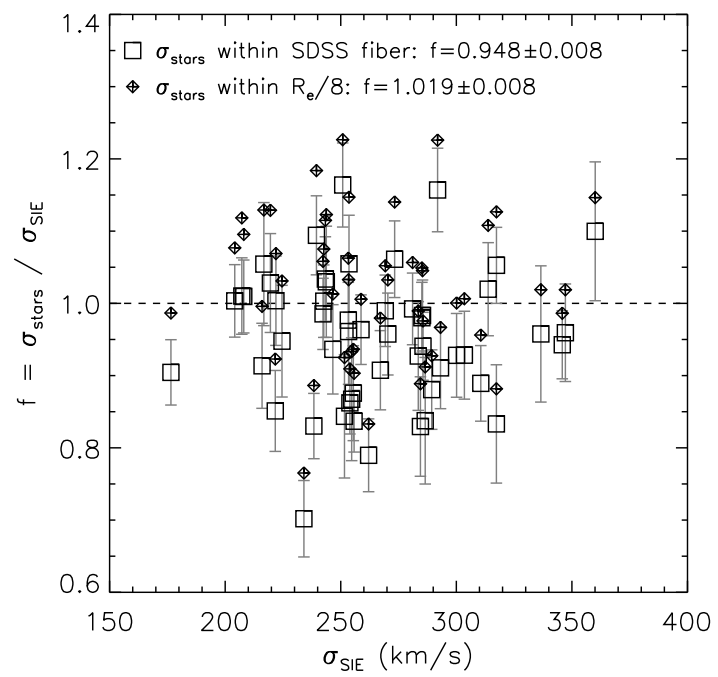

FIG. 1.- Ratio $f$ of stellar velocity dispersion $\sigma_{\text {stars }}$ to velocitydispersion parameter $\sigma_{\mathrm{SIE}}$ of the best-fit lens model, as a function of $\sigma_{\mathrm{SIE}}$. Points are shown for $\sigma_{\text {stars }}$ as observed within the $3^{\prime \prime}$-diameter SDSS fiber, and as corrected to a uniform physical aperture of $R_{e} / 8$ using the empirical formula of Jørgensen et al. (1995). For visual clarity, error bars (gray) are only plotted for the fiber-aperture points.

logarithmic mass-profile slopes (from a combined lensing and dynamical analysis) and the number of near neighbor galaxies to the lenses. The (small) intrinsic scatter of the individual mass slopes about the average value is directly related to the scatter in the $\sigma_{e 8}-\sigma_{\mathrm{SIE}}$ relation; it remains to be determined whether the Auger (2007) result is confirmed by the larger current SLACS sample (Treu et al., in preparation).

\section{THE FUNDAMENTAL PLANE AND MASS PLANE}

The fundamental plane (FP: Diorgovski \& Davis 1987; Dressler et al. 1987) is the name given to the approximately two-dimensional manifold defined by early-type galaxies within the three-dimensional space spanned by the logarithms of effective radius $R_{e}$, surface brightness $I_{e}$, and central velocity dispersion $\sigma_{c}$. When the $\mathrm{FP}$ is expressed in the form

$$
\log _{10} R_{e}=a \log _{10} \sigma_{c}+b \log I_{e}+d,
$$

the coefficients $a$ and $b$ are found to be in the approximate ranges 1.1 to 1.6 and -0.75 to -0.8 respectively, depending upon the sample, wave-band, and methods of observation and fitting (see Tables 2 and 4 of Bernardi et al. 2003). As has been discussed extensively in the literature, the implications of this relationship can be understood in terms of a simple dimensional analysis of the form

$$
R_{e}=c G^{-1} \sigma_{c}^{2} \Upsilon^{-1} I_{e}^{-1},
$$

where $\Upsilon$ is the total mass-to-light ratio within some physical aperture and $c$ is a dimensionless constant parameterizing the details of mass-dynamical structure. Equation 2 with the values $(a, b)=(2,-1)$ is often referred to as the "virial plane", and the observational fact that $a<2$ and $b>-1$ is referred to as the "tilt" of the FP relative to the virial plane. The terminology is perhaps misleading: the tilt of the FP does not imply a lack of virial equilibrium in the individual galaxies that define 
the plane; it simply requires that $c$ and/or $\Upsilon$ vary as a function of the other observables.

B07 constructed the FP of SLACS lenses and showed that the SLACS FP coefficients were consistent with those of the larger SDSS early-type galaxy population, while Paper II showed consistency between the SLACS lenses and the FP of local galaxies, corrected for luminosity evolution. We now revisit the calculation of B07 for the larger current sample of lenses (53 early-type lens galaxies with well-measured SDSS velocity dispersions), using the data presented in Paper V. ${ }^{11}$ We describe the FP in the form of Equation 22 with $\sigma_{e 2}$ taking the role of central velocity dispersion $\sigma_{c}$. As noted above, the aperture of $R_{e} / 2$ is chosen to reflect the approximate median ratio of Einstein radius $R_{\text {Ein }}$ to $R_{e}$ within the sample. Due to the velocity-dispersion aperture correction formula that we employ (Jørgensen et al. 1995), the $\sigma_{e 2}$ values are related trivially to the more commonly quoted $\sigma_{e 8}$ values by a fixed factor, with $\log _{10} \sigma_{e 8}=\log _{10} \sigma_{e 2}+0.0241$. The best-fit FP coefficients as determined by the method described in $\$ 2$ are presented in Table 2. The plane is shown in edge-on projection in the left panel of Figure 2. The RMS residual scatter in $\log _{10} R_{e}$ of the fit is 0.064 dex. The RMS error-scaled orthogonal residual scatter of the fit is 1.44, reasonably consistent with error estimates but indicating a degree of intrinsic scatter. The best-fit coefficients are consistent with those determined by Bernardi et al. (2003) from SDSS data for a larger early-type galaxy sample, though the details of that analysis are somewhat different than ours.

As originally noted in B07, there is a significant correlation between log-radius residuals about the best-fitting FP and the rest-frame $V$-band mass-to-light ratio $\Upsilon_{V}$ (for mass and light within $R_{e} / 2$, evaluated from B-spline light models and SIE mass models). This correlation has a linear coefficient $r=-0.337$ (corresponding to a $98.6 \%$ significance) and is in the sense that lens galaxies with effective radii larger than their FP-predicted values tend to have lower mass-to-light ratios. This is to be expected from a simple dimensional analysis as in Equation 3 if $\sigma$ and $I$ are held fixed (i.e., for a given point on the FP), an increase in $R$ should give a decrease in $\Upsilon_{V}$. This correlation suggests observationally that the scatter about the FP is due at least in part to an intrinsic scatter in mass-to-light ratios at a given point in the plane.

In addition to the FP, we consider the "mass plane" (MP) of SLACS lenses as defined in B07. By using strong-lensing aperture masses (corrected to the uniform aperture of $R_{e} / 2$ ), we can replace the surface brightness $I_{e}$ with the surface mass density $\Sigma_{e 2}$ within $R_{e} / 2$. The MP formalism is attractive in that it is independent of any luminosity-evolution effects that occur in a spatially uniform manner within the lens galaxies. Thus the MP can be tracked across redshift without correction for the dimming of stellar luminosity. The MP should also be better suited to comparison with theory and numerical simulation of galaxy formation, merging, and dynamical evolution processes. Since the scale length of the MP is still taken from the luminosity distribution and the MP

\footnotetext{
11 Our current determination also differs from that of B07 in the use of luminosities based upon de-bugged $k$-corrections as described in Paper V. The overall conclusions of B07 are unchanged.
}

TABLE 2

FP AND MP COEFFICIENTS

\begin{tabular}{cccr}
\hline \hline Surface & & & \\
Density & $a$ & $b$ & $d$ \\
\hline$I_{e}$ & $1.28 \pm 0.22$ & $-0.77 \pm 0.07$ & $-0.09 \pm 0.07$ \\
$\Sigma_{e 2, \mathrm{SIE}}$ & $1.82 \pm 0.19$ & $-1.20 \pm 0.12$ & $0.91 \pm 0.14$ \\
$\Sigma_{e 2, \mathrm{LTM}}$ & $2.10 \pm 0.24$ & $-0.83 \pm 0.12$ & $0.56 \pm 0.15$ \\
\hline
\end{tabular}

NOTE. - Coefficients are for FP and MP relations in the form $\log _{10} R_{e}=a \log _{10} \sigma_{e 2}+b \log _{10}$ (surface density) $+d . R_{e}$ measured in kpc, $\sigma_{e 2}$ in units of $100 \mathrm{~km} \mathrm{~s}^{-1}, I_{e}$ in units of $10^{9} L_{V, \odot} / \mathrm{kpc}^{-2}$, and $\Sigma_{e 2}$ in units of $10^{9} \mathrm{M}_{\odot} / \mathrm{kpc}^{-2}$. Units are chosen so as to reduce artificial covariance between plane coefficients and plane zero-points. Fit method is described in $\$ 2$

velocities are still traced by the stars, it is not a pure mass space formulation. However, one can extract total (luminous plus dark matter) aperture masses, stellar effective radii, and stellar velocity dispersions from any sensibly constructed theory of galaxy formation, evolution, and merging. One cannot extract luminosities without delving into the entirely different domain of stellar populations and their evolution. Thus the MP formulationand more elementally, the data upon which it is basedrepresent an important step in bringing theory and observation together by separating the subject of stellar populations from the subject of galaxy formation.

We express the MP in a form analogous to the FP:

$$
\log _{10} R_{e}=a_{m} \log _{10} \sigma_{e 2}+b_{m} \log _{10} \Sigma_{e 2}+d_{m} .
$$

Since $\Sigma_{e 2}$ depends somewhat upon the lens model used to evaluate the surface density, we fit for MP coefficients using $\Sigma_{e 2}$ values from both the SIE (singular isothermal ellipsoid) and LTM (light-traces-mass) mass models of Paper V. Values for the best-fit coefficients are given in Table 2. The best-fit MP (using the SIE aperture masses) is seen in edge-on projection in the right-hand panel of Figure 2, The RMS residual scatter in $\log _{10} R_{e}$ about the best-fit MP is 0.076 dex using SIE surface densities and 0.10 dex using LTM surface densities. The RMS error-scaled orthogonal residuals are 1.24 (SIE) and 1.50 (LTM). For the case of the SIE-based MP, the plane is tighter than the FP when judged by the RMS errorscaled orthogonal residuals (the quantity minimized in the fitting), but not exceptionally so: the edge-on MP is not noticeably tighter than the edge-on FP as seen in of Figure 2.

We can see from Table 2 that the coefficients of the $\mathrm{MP}$ are in a sense less "tilted" relative to the values $\left(a_{m}, b_{m}\right)=(2,-1)$. To quantify this, we consider $10^{6}$ bootstrap re-samplings of our lens sample, from which we compute confidence limits in the $a_{m}-b_{m}$ plane. The values $\left(a_{m}, b_{m}\right)=(2,-1)$ fall on the contour enclosing $77 \%$ $(60 \%)$ of the re-sample coefficient points for SIE (LTM) based fits, showing that the MP is in fact fairly consistent with the plane represented by $\left(a_{m}, b_{m}\right)=(2,-1)$. We cast this statement in more physical terms in 6.

\section{LENSING MASS, DYNAMICAL MASS, AND LUMINOSITY}

Depending on the details of the coefficients, the FP can be understood in terms of a systematic relation between luminosity and a dimensional mass variable ${ }^{12}$ such

\footnotetext{
12 Our "dimensional mass" $M_{\text {dim }}$ is, modulo a constant factor,
} equal to the "effective mass" or "dynamical mass" referred to by 

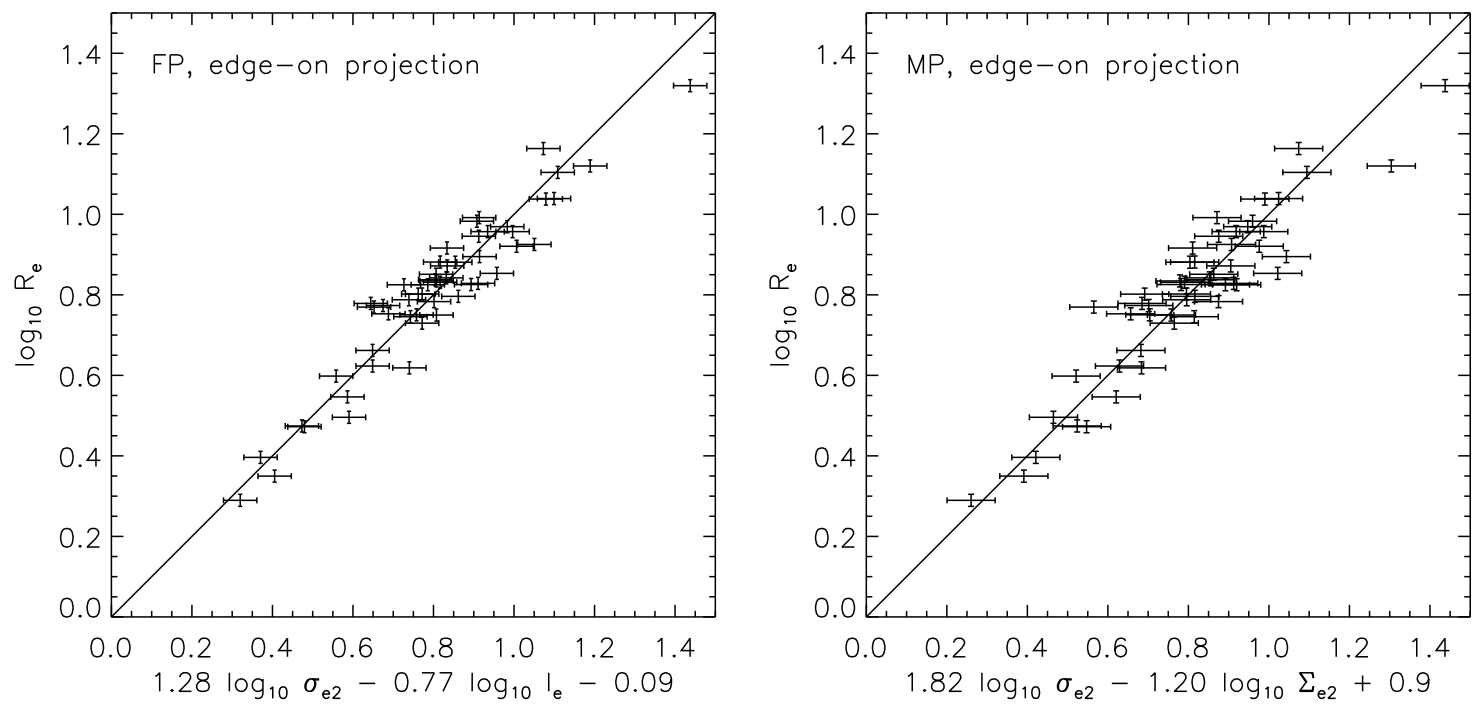

FIG. 2.- Edge-on projections of the best-fit FP (left) and MP (right) relationships for the SLACS lens sample. Units are $100 \mathrm{~km} \mathrm{~s}^{-1}$ for $\sigma_{e 2}, 10^{9} L_{V, \odot} \mathrm{kpc}^{-2}$ for $I_{e}, 10^{9} M_{\odot} \mathrm{kpc}^{-2}$ for $\Sigma_{e 2}$, and $\mathrm{kpc}$ for $R_{e}$. These are the same units used in the FP and MP fits, and were chosen so as to lessen covariance between plane coefficients and plane zero-point. Diagonal solid lines represent equality between $\log _{10} R_{e}$ as observed and as predicted from the best-fit FP.

as $M_{\text {dim }} \equiv G^{-1} \sigma_{e 2}^{2}\left(R_{e} / 2\right)$ of the form $L \propto M_{\text {dim }}^{\eta}$ (e.g., Faber et al. 1987; Bender et al. 1992; Ciotti et al. 1996). Such a relation leads to FP coefficients in Equation 2 given by $a=2 \eta /(2-\eta)$ and $b=-1 /(2-\eta)$. This may be conceptualized as a systematic variation of a "dimensional mass-to-light ratio" $\Upsilon_{\operatorname{dim}} \equiv M_{\operatorname{dim}} / L$ with $M_{\operatorname{dim}}$. It is, however, important to keep in mind that $\Upsilon_{\text {dim }}$ is linearly proportional to the true mass-to-light ratio $\Upsilon$ only if the FP tilt is due to a systematic mass-to-light ratio trend, rather than to a trend in mass-dynamical structure.

The availability of strong-lensing aperture masses in addition to the traditional FP observables allows us to directly test the alternative hypotheses for the "tilt" of the FP, because in addition to $M_{\text {dim }}$, we measure the aperture mass $M_{\text {lens }}$ from strong lensing. Consider the following two relations:

$$
\begin{aligned}
L_{V} & =c_{1} M_{\mathrm{dim}}^{\eta}, \\
M_{\text {lens }} & =c_{0} M_{\mathrm{dim}}^{\delta} .
\end{aligned}
$$

Consider also the following two alternative hypotheses: (1) on average, early-type galaxies have a universal massdynamical structure, and the tilt of the FP is due to a systematic trend in total mass-to-light ratio with mass; or (2) on average, early-type galaxies have a universal mass-to-light ratio, and the tilt of the FP is due to a systematic trend in mass-dynamical structure. If hypothesis (1) is correct, then we should find $\delta \simeq 1$, whereas if hypothesis (2) is correct, we should find $\delta \simeq \eta$. Of course, we may also find that $\eta<\delta<1$, since in principle both explanations could contribute to the tilt of the FP. Roughly speaking, hypothesis (1) represents "homology" and hypothesis (2) represents "non-homology".

Before proceeding, we note that numerous works have explored the possible role of a systematically varying Sérsic index $n$ in causing the tilt of the FP

other authors. We use the term "dimensional" to avoid confusion. (e.g., Hiorth \& Madsen 1995; Graham \& Colless 1997; Bertin et al. 2002; Trujillo et al. 2004), and thus we must not disregard this possibility in our own analysis. We compute $n$ for all SLACS lenses by continuing the de Vaucouleurs model optimizations after freeing the index from its fixed $n=1 / 4$ value. We find that $n$ is completely uncorrelated with lensing mass, dynamical mass, luminosity, and velocity dispersion within the sample, and therefore the inclusion of the Sérsic index as a significant factor in our analysis is not motivated by the data. This lack of correlation between $n$ and other quantities is in fact consistent with other studies, since the SLACS sample is confined to relatively high-mass/highluminosity early-type galaxies, and does not extend over a sufficient range to define the $n-L$ correlation significantly given the level of intrinsic scatter (e.g., Caon et al. 1993; D’Onofrio et al. 1994; Graham \& Guzmán 2003; Ferrarese et al. 2006).

Table 3 shows the results of fits for the normalizations and exponents of the relations defined in Equations 5 and 6. as well as for the relation between luminosity and lensing mass. Within the uncertainties, the clear result is that $\delta \simeq 1$ while $\eta<1$ : thus, we conclude based upon our sample of lenses that the tilt of the FP — as defined by massive ellipticals - is due to a systematic trend in massto-light ratio and not to a trend in mass-dynamical structure. These relations are shown graphically in Figure 3. where we can see by eye that the logarithmic slope of the $M_{\text {lens }}$-versus- $M_{\text {dim }}$ relation is significantly less shallow than those of the $L_{V}$-versus- $M_{\operatorname{dim}}$ and $L_{V}$-versus- $M_{\text {lens }}$ relations. The slopes of the $L$-versus- $M$ relations change only negligibly when the de Vaucouleurs model luminosity is replaced with the more general radial B-spline model aperture luminosity within $R_{e} / 2$ (described in $\mathrm{Pa}-$ per $\mathrm{V})$. If we fix $\delta=1$, we find $\left\langle\log _{10}\left[M_{\text {lens }} / M_{\operatorname{dim}}\right]\right\rangle=$ $0.530 \pm 0.012$ (RMS of 0.08 ) for SIE aperture corrections and $\left\langle\log _{10}\left[M_{\text {lens }} / M_{\text {dim }}\right]\right\rangle=0.543 \pm 0.014$ (RMS of 0.1$)$ for LTM corrections. Accounting for our best estimates of the typical measurement errors, the intrinsic scatter 
in $\log _{10}\left[M_{\text {lens }} / M_{\text {dim }}\right]$ about the mean value that gives a reduced $\chi^{2}$ of approximately unity is 0.057 dex RMS, or about a $\pm 13 \%$. This is consistent with the intrinsic velocity-dispersion scatter of $7.5 \%$ seen in $\$ 4$ given that $M_{\text {dim }}$ scales as the square of velocity dispersion.

The result that $\delta \simeq 1$ while $\eta<1$ is one of the central results of this work and of B07, and thus its significance merits special attention. Specifically, we consider the distribution of pairs of $\eta$ and $\delta$ values fitted to the same sets of bootstrap samples. For both the SIE and LTM aperture masses, we generate $10^{6}$ bootstrap re-samples and fit $\eta$ and $\delta$ for each. The resulting distributions can be seen in Figure 4. We see that for both mass models, $\delta$ is significantly greater that $\eta$ and consistent with $\delta=1$, while $\eta=1$ and $\delta=\eta$ are ruled out at high significance.

The skeptic might worry that our result of a universal mass-density structure as a function of mass has been "baked in the cake" by our use of a single universal profile (i.e., the isothermal model or the approximately de Vaucouleurs LTM model) to evaluate aperture masses for the uniform physical aperture of $R_{e} / 2$. The robustly measured quantity is the mass within the Einstein radius $R_{\text {Ein }}$ (Kochanek 1991), and in general $R_{\text {Ein }}$ differs from $R_{e} / 2$ (though, by design, not by a large amount). If such an explanation were masking a true mass-dependent trend in structure, we would expect to see a correlation of $R_{\text {Ein }} / R_{e}$ with the residuals about the best-fit $M_{\text {lens }^{-}}$ versus- $M_{\text {dim }}$ relationship. In fact, we see no such correlation. In addition, if we restrict our analysis to that half of the lens sample for which $R_{\text {Ein }}$ is most nearly matched to $R_{e} / 2$ (as quantified by the fractional difference between the aperture masses within $R_{\operatorname{Ein}}$ and $R_{e} / 2$ ), we see no significant changes in our results.

We can easily translate our relations between $V$-band luminosity and lensing mass within $R_{e} / 2$ (the last two rows of Table 3) into expressions for the mass-to-light ratio within $R_{e} / 2$ as a function of luminosity. Using the fact that $32.0 \%$ of the total de Vaucouleurs model flux is enclosed within the $R_{e} / 2$ aperture, we have

$$
\frac{\Upsilon_{V, e 2}}{\Upsilon_{V, \odot}}=c_{2}^{-1 / \eta^{\prime}}\left(\frac{1}{0.320}\right)\left(\frac{L_{V}}{L_{V, \odot}}\right)^{\left[\left(1 / \eta^{\prime}\right)-1\right]} .
$$

For SIE aperture masses, we find $\log _{10}\left(\Upsilon_{V, e 2} / \Upsilon_{V, \odot}\right)=$ $(0.83 \pm 0.01)+(0.37 \pm 0.06) \log _{10}\left(L_{V} / 10^{11} L_{V, \odot}\right)$, while for LTM aperture masses we obtain $\log _{10}\left(\Upsilon_{V, e 2} / \Upsilon_{V, \odot}\right)=$ $(0.83 \pm 0.01)+(0.29 \pm 0.05) \log _{10}\left(L_{V} / 10^{11} L_{V, \odot}\right)$-i.e., a total central $V$-band mass-to-light ratio of approximately 6.8 times solar for a $V$-band luminosity of $10^{11}$ times solar. This $\Upsilon-L$ relation should not necessarily be regarded as fundamental, however: we find comparably significant correlations of $\Upsilon$ with $M_{\text {lens }}, M_{\mathrm{dim}}, R_{e}$, and $\sigma_{e 8}$.

The details of our mass-luminosity relations are sensitive to the assumed rate of passive evolution which we have attempted to remove from the sample. The SLACS lens sample exhibits a significant luminosity-redshift degeneracy (see Paper V) which prevents us from solving simultaneously for the mass and redshift dependence of luminosity. The main effect that we expect is a certain level of unmodeled luminosity dependence in the rate of $V$-band evolution, characteristic of more recent star formation (and hence faster fading) in less luminous galaxies (e.g., Cowie et al. 1996; Treu et al. 2005a,b; van der Wel et al. 2006), though the effect should be less pronounced in the $V$ band than in the $B$ band. In the absence of published constraints on the mass dependence of $V$-band luminosity evolution, we consider the effects of a simple "toy model" whereby the rate of luminosity evolution varies from $d \log _{10} L_{V} / d z=0.6$ to 0.2 linearly over the range $\log _{10}\left(M_{\text {lens }} / M_{\odot}\right)=10$ to 12 . (This is likely to be an extreme scenario - see the rest-frame $B$-band analysis of Treu et al. 2005b.) In this case, the exponents of the $L$-versus- $M$ relations given in Table 3 change from $\eta=0.77$ to $\eta=0.82$ and $\eta^{\prime}=0.73$ to $\eta^{\prime}=0.78$, with unchanged uncertainties. We defer more detailed analysis until the completion of multi-band observations currently underway with $H S T$-WFPC2, which will enable the determination of rest-frame $B$-band luminosities and subsequent connection to a more comprehensive literature on luminosity evolution (e.g, Treu et al. 2002, 2005b; van der Wel et al. 2005; di Serego Alighieri et al. 2005). These ambiguities of luminosity evolution have no bearing upon the pure mass-dynamical relations, and no effect upon our result that the structure of massive elliptical galaxies is (on average) independent of galaxy mass.

\section{ROBUST ENSEMBLE MEASUREMENT OF THE RADIAL MASS-DENSITY PROFILE}

In this section, we constrain the average projected mass-density profile of our lens galaxies without any dynamical modeling by considering the lens ensemble as a whole, and using the fact that the aperture masses are measured most robustly for physical apertures (i.e., Einstein radii) ranging from 0.2 to 1 times the effective radius depending upon the individual lens. ${ }^{13}$ Motivated by the FP, we regard the sample as a twoparameter family, parameterized by effective radius $R_{e}$ and velocity dispersion $\sigma_{e 2}$. From these quantities, we construct the previously defined "dimensional mass" $M_{\text {dim }} \equiv G^{-1} \sigma_{e 2}^{2}\left(R_{e} / 2\right)$. We then scale the measured aperture masses within the Einstein radii $M(<R)=$ $M_{\text {Ein }}$ by $M_{\text {dim }}$, and scale the corresponding apertures $R=R_{\text {Ein }}$ by the effective radii $R_{e}$. The resulting dimensionless projected mass-radius relation is shown in logarithmic coordinates in Figure 5. Describing this relation with a power law (i.e., linear in the logarithmic coordinates) in the form

$$
\log _{10}\left[M(<R) / M_{\mathrm{dim}}\right]=g \log _{10}\left[R / R_{e}\right]+h,
$$

we find $g=1.10 \pm 0.09$ and $h=0.85 \pm 0.03$ for SIE aperture masses or $g=1.13 \pm 0.11$ and $h=0.85 \pm 0.03$ for LTM aperture masses. RMS orthogonal residuals scaled by error estimates are 1.27 (SIE) and 1.41 (LTM), indicating reasonable consistency of the data with the fitted relation, but with evidence for intrinsic scatter as in other relations. The residuals about the bestfit relation are completely uncorrelated with $M_{\mathrm{dim}}$, an echo of the mass-independent structure result of $₫ 6$. For a pure singular isothermal model, the projected aperture mass scales as $M(<R) \propto R$-i.e., $g=1$. Thus

13 In fact, the sample probes three-dimensional radii even beyond the physical scale of $R_{e}$, due to the sensitivity of lensing to projected mass. For the singular isothermal sphere, $36 \%$ of the mass (the exact fraction is $1-2 / \pi$ ) within a cylinder of radius $R$ is exterior to the corresponding sphere of the same radius. 
TABLE 3

MASS AND LIGHT SCALING RELATIONS

\begin{tabular}{lcc}
\hline \hline Scaling Relation & Prefactor & Exponent \\
\hline$\left(M_{\mathrm{SIE}} / 10^{11} M_{\odot}\right)=c_{0}\left(M_{\operatorname{dim}} / 10^{11} M_{\odot}\right)^{\delta}$ & $\log _{10} c_{0}=0.54 \pm 0.02$ & $\delta=1.03 \pm 0.04$ \\
$\left(M_{\mathrm{LTM}} / 10^{11} M_{\odot}\right)=c_{0}\left(M_{\operatorname{dim}} / 10^{11} M_{\odot}\right)^{\delta}$ & $\log _{10} c_{0}=0.54 \pm 0.02$ & $\delta=0.99 \pm 0.05$ \\
$\left(L_{V} / 10^{11} L_{\odot}\right)=c_{1}\left(M_{\operatorname{dim}} / 10^{11} M_{\odot}\right)^{\eta}$ & $\log _{10} c_{1}=0.16 \pm 0.02$ & $\eta=0.77 \pm 0.04$ \\
$\left(L_{V} / 10^{11} L_{\odot}\right)=c_{2}\left(M_{\mathrm{SIE}} / 10^{11} M_{\odot}\right)^{\eta \prime}$ & $\log _{10} c_{2}=-0.24 \pm 0.01$ & $\eta^{\prime}=0.73 \pm 0.03$ \\
$\left(L_{V} / 10^{11} L_{\odot}\right)=c_{2}\left(M_{\mathrm{LTM}} / 10^{11} M_{\odot}\right)^{\eta \prime}$ & $\log _{10} c_{2}=-0.26 \pm 0.01$ & $\eta^{\prime}=0.77 \pm 0.03$ \\
\hline
\end{tabular}

Note. - Mass values $M_{\mathrm{SIE}}$ and $M_{\mathrm{LTM}}$ are masses within $R_{e} / 2$ as evaluated from SIE and LTM lens models. Dimensional mass variable is defined as $M_{\mathrm{dim}} \equiv G^{-1} \sigma_{e 2}^{2}\left(R_{e} / 2\right)$. Fit method is described in
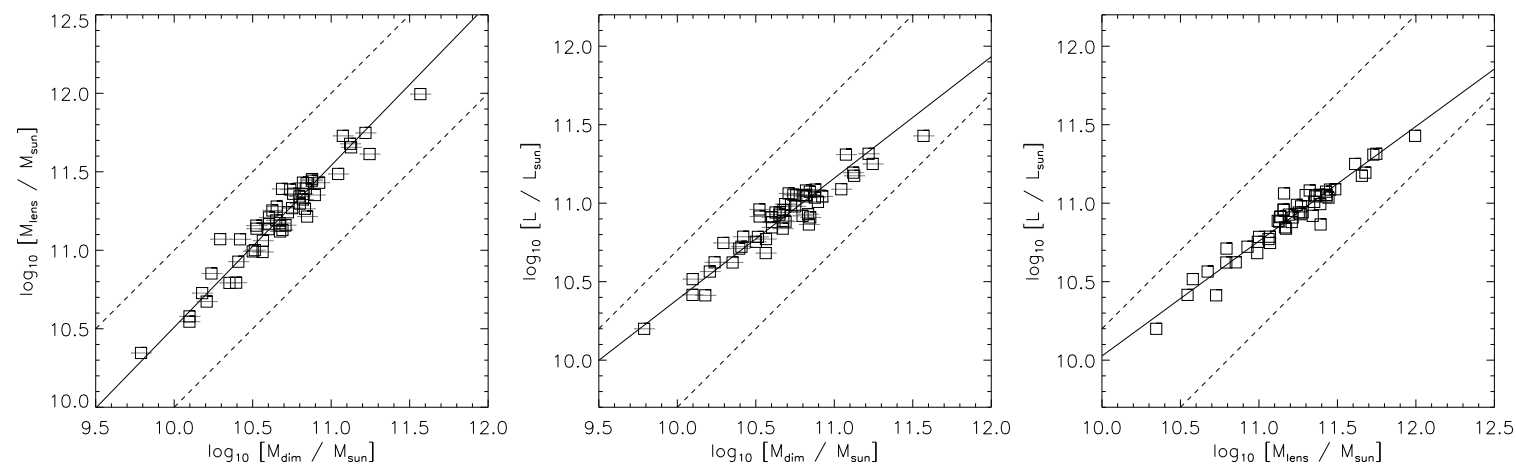

FIG. 3.- Relationships between mass and luminosity variables. Left panel: Lensing mass $M_{\text {lens }}$ within one-half effective radius (evaluated from the SIE lens models) versus dimensional mass variable $M_{\operatorname{dim}}=G^{-1} \sigma_{e 2}^{2}\left(R_{e} / 2\right)$. Center panel: Rest-frame $V$-band luminosity $L$ versus $M_{\text {dim. }}$. Right panel: Rest-frame $V$-band luminosity $L$ versus $M_{\text {lens. }}$ In each panel, the best-fit linear relationship between the two logarithmic quantities is shown by the solid line. Dashed lines indicate a slope of unity, for reference. Typical errors in $M_{\text {dim }}$ are shown by gray error bars in the left and center panels; errors in $M_{\text {lens }}$ and $L$ are smaller than the plot symbols.

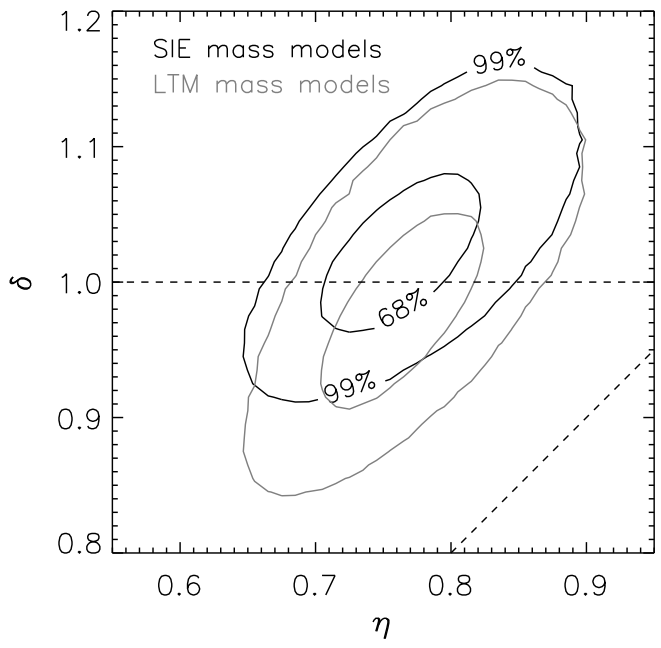

Fig. 4.- Distribution of $\eta$ and $\delta$ parameter fits to $10^{6}$ bootstrap re-samples. Results are shown for lensing aperture masses evaluated using both SIE lens models (black) and LTM lens models (gray). Dashed lines of $\delta=1$ and of $\delta=\eta$ are shown as well.

we find that our data are consistent with the universal total-mass profile being isothermal. Translating back into a three-dimensional power-law profile, our results correspond to $\rho(r) \propto r^{-1.90 \pm 0.09}$ (using SIE aperture masses) and $\rho(r) \propto r^{-1.87 \pm 0.10}$ (using LTM aperture masses). This analysis is very similar to that employed by Rusin et al. (2003) and Rusin \& Kochanek (2005) to derive constraints on the quasar-lens population. The key difference for our application to SLACS is that, since we have stellar velocity dispersions for our entire sample, we can directly scale lensing masses by dynamical masses, ignoring issues of luminosity trends and evolution rates. The most likely average power-law profile found by Rusin \& Kochanek (2005) is slightly steeper $\left(\rho(r) \propto r^{-2.06 \pm 0.17}\right)$ than that found in this work, but the two results are consistent within their combined errors. Our (nearly) isothermal result is also consistent with the lens+dynamical modeling results of LSD and Paper III, with the dynamical analysis of Gerhard et al. 2001, and with the strong+weak lensing analysis of Paper IV. The particular advantage of the method presented here is that it is simple and robust, with little susceptibility to assumptions or systematic errors. (e.g., Gerhard et al. 2001; Rusin et al. 2003; LSD; Paper III; Paper IV).

For comparison, the logarithmic slope of the projected enclosed luminosity profile changes very little over the range 0.3 to 1 times $R_{e}$ where the bulk of our data are concentrated, having a value of $g_{\text {light }}=0.71$ for the de Vaucouleurs model and a mean value of $g_{\text {light }}=0.68$ for the more general B-spline luminosity profile models of Paper V. If a light-traces-mass model were appropriate, we would expect to find similar values for the logarithmic slope of enclosed mass with radius. The onesided $99 \%, 99.9 \%$, and $99.99 \%$ lower limits on the mass slope variable $g$ from bootstrap re-samples are $0.85,0.76$, and 0.67 respectively for SIE fits $(0.83,0.72$, and 0.62 for LTM fits). Thus with only three basic observablesvelocity dispersion, effective radius, and Einstein radius (as translated into physical units and enclosed mass using 


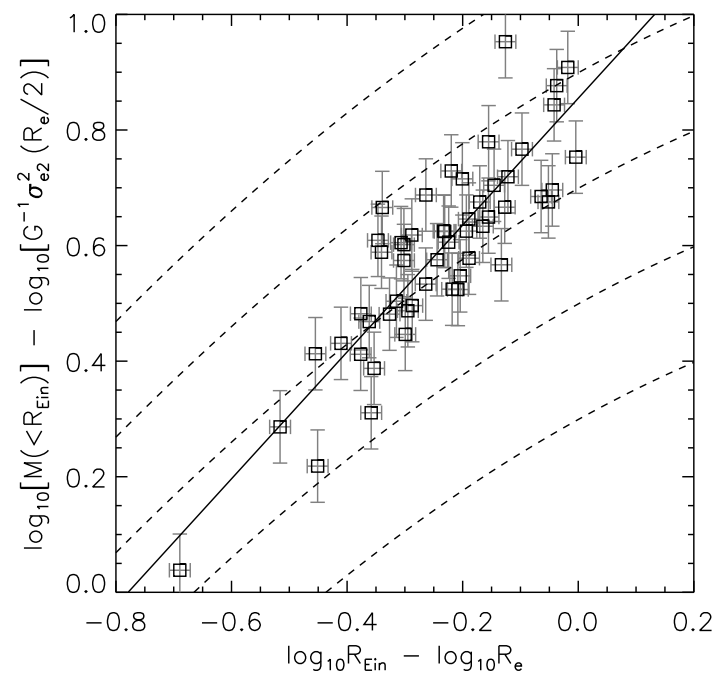

FIG. 5.- Dimensionless projected mass-radius relation. Horizontal axis is mass-aperture (Einstein) radius scaled by lens-galaxy effective radius. Vertical axis is lensing aperture mass within the Einstein radius scaled by the dimensional mass variable formed from the combination of central velocity dispersion and effective radius. Error bars show the typical error in each coordinate used in the scaled orthogonal fit for the best relation. The solid line shows this best-fit linear relation in the logarithmic coordinates, given by $\log _{10}\left[M(<R) / M_{\mathrm{dim}}\right]=(1.10 \pm 0.09) \log _{10}\left[R / R_{e}\right]+(0.85 \pm$ $0.03)$. For comparison, dashed lines show the logarithmic enclosedluminosity profile for the de Vaucouleurs surface-brightness model, with various offsets. If a light-traces-mass model were correct, the data points would trace the slope of these de Vaucouleurs curves.

spectroscopic redshifts) - and no dynamical modeling, we can falsify the light-traces-mass hypothesis at $99.9 \%$ to 99.99\% confidence even inside the effective radius, where dark matter is often assumed to play little role. This result argues for a preference of SIE lens model parameters over those from the LTM models. Figure 5 shows the slope of the de Vaucouleurs aperture-luminosity profile for comparison with the enclosed-mass data points; the inconsistency of the data with an LTM model is visually apparent.

We can translate our measurement into a lower limit on the average central dark-matter fraction by making a "maximal bulge" assumption - specifically, assuming that the total mass and the stellar mass are equal within $0.3 R_{e}$. We then take the projected stellar mass-profile to go as $M_{\text {stars }}(<R) \propto R^{0.7}$ (following the luminosity profile) and the projected total-mass profile to go as $M_{\text {total }}(<R) \propto R^{1.1 \pm 0.1}$. This gives a lower limit on the average projected dark-matter fraction of $f_{\mathrm{DM}}=$ $0.38 \pm 0.07$ within one effective radius $R_{e}$. This is consistent with the highest values found through dynamical analysis of nearby elliptical galaxies (e.g., Gerhard et al. 2001).

\section{SUMMARY OF RESULTS}

By combining the direct mass measurements provided by the strong gravitational lensing effect with traditional measures of galaxy luminosity, size, and velocity dispersion, we have derived empirical scaling laws for massive early-type galaxies. The results of our analyses are summarized as follows. We remind the reader that these results apply specifically to galaxies with velocity dispersions between approximately 175 and $400 \mathrm{~km} \mathrm{~s}^{-1}$, rest- frame $V$-band luminosities between $10^{10}$ and $3 \times 10^{11} L_{\odot}$, and redshifts between 0.06 and 0.36. (The few higher redshift SLACS lenses presented in Paper $\mathrm{V}$ have an SDSS spectral SNR that is too low for a reliable velocitydispersion measurement.)

1. The projected major axes of the mass and light distributions within the SLACS lens sample are aligned to $\langle\triangle P A\rangle=2.5^{\circ} \pm 2.4^{\circ}\left(\mathrm{RMS}\right.$ of $\left.18^{\circ}\right)$ for 55 lenses with light axis ratios $q_{\text {stars }}<0.95$. Restricted to the subset of 29 lenses whose angular structure is tightly constrained by lensed images through an extended azimuthal range about the lens center, this alignment tightens to $\langle\triangle P A\rangle=$ $1.4^{\circ} \pm 1.9^{\circ}$, with an RMS of $10^{\circ}$.

2. For light-traces-mass (LTM) lens models, the required external shears are preferentially aligned with the major axes of the lens galaxies at extremely high significance when the sample as a whole is considered. This alignment suggests a falsification of the LTM hypothesis.

3. The average relative flattening between mass and light for the SLACS sample, measured by the ratio of projected (2D) minor-to-major axis ratios $q_{\mathrm{SIE}}$ (mass) and $q_{\text {stars }}$ (light), is $\left\langle q_{\text {SIE }} / q_{\text {stars }}\right\rangle=1.02 \pm$ 0.02 (RMS of 0.12 ): i.e., consistent with unity on average. We see a decreasing trend in $q_{\mathrm{SIE}} / q_{\mathrm{stars}}$ with increasing velocity dispersion $\sigma_{\text {SIE }}$ (measured from the SIE lens models).

4. The ratio $f \equiv \sigma_{e 8} / \sigma_{\mathrm{SIE}}$ of central stellar velocity dispersions to isothermal lens-model velocity dispersions is $f=1.019 \pm 0.008$. The RMS scatter about the mean is approximately 0.1 , which when considered along with measurement errors corresponds to an intrinsic velocity scatter of about $20 \mathrm{~km} \mathrm{~s}^{-1}$ (or about $7.5 \%$ of $\sigma_{\text {SIE }}$ ). The residual scatter about the mean relation is not correlated with mass, luminosity, velocity dispersion, massto-light ratio, effective radius, or ratio of Einstein radius to effective radius.

5. The SLACS lens galaxies define a fundamental plane (FP) that is consistent with the FP measured for the general population of early-type galaxies observed by the SDSS and other surveys.

6. The SLACS lens sample also defines a "mass plane" (MP) relation, obtained by replacing surface brightness with surface mass density as measured from strong lensing. The MP is significantly less "tilted" than the FP, and is essentially consistent with simple expectations based on the virial theorem and a universal mass-dynamical structure within the population.

7. The relationship between rest-frame $V$ band luminosity $L_{V}$ and the dimensional mass variable $M_{\mathrm{dim}} \equiv G^{-1} \sigma_{e 2}^{2}\left(R_{e} / 2\right)$ is given by $\log _{10}\left[L_{V} / 10^{11} L_{\odot}\right]=(0.77 \pm$ $0.04) \log _{10}\left[M_{\mathrm{dim}} / 10^{11} M_{\odot}\right]+(0.16 \pm 0.02)$. A similar relation holds when $M_{\text {dim }}$ is replaced with the strong lensing-determined 
mass within $R_{e} / 2: \quad \log _{10}\left[L_{V} / 10^{11} L_{\odot}\right]=$ $(0.73 \pm 0.03) \log _{10}\left[M_{\text {lens }, e 2} / 10^{11} M_{\odot}\right]-(0.24 \pm 0.01)$. The consistency between the slopes of these two relations implies that the systematic "dynamical" mass-to-light variation inferred from the $\mathrm{FP}$ is representative of a true mass-to-light variation within the early-type galaxy population.

8. The relationship between $M_{\mathrm{dim}}$ and the mass $M_{\text {lens }}$ within $R_{e} / 2$ as measured by strong lensing is given by $\log _{10}\left[M_{\text {lens }} / 10^{11} M_{\odot}\right]=(1.03 \pm$ $0.04) \log _{10}\left[M_{\operatorname{dim}} / 10^{11} M_{\odot}\right]+(0.54 \pm 0.02)$. The unitary (within errors) slope of this $M$-versus- $M$ relation indicates that the mass-dynamical structure of early-type galaxies does not vary systematically with mass over the range probed by the SLACS sample (approximately $9.8<\log _{10}\left[M_{\operatorname{dim}} / M_{\odot}\right]<$ 11.6). Our result indicates that the dimensional mass is a suitable proxy for the true mass within the central regions of massive early-type galaxies. Fitting directly for an overall scaling between lensing and dimensional masses, we find that $\left\langle\log _{10}\left[M_{\text {lens }} / M_{\text {dim }}\right]\right\rangle=0.530 \pm 0.012$ with an RMS scatter of 0.08 dex about the mean. After accounting for measurement errors, this scatter is approximately $0.057 \mathrm{dex}$ (or about $\pm 13 \%$ ).

9. The difference between the slopes of the $M-L$ relations of result [7] and the $M-M$ relation of result 8 is very highly significant, and implies that the "tilt" of the FP is due to a systematic variation in the total mass-to-light ratio with mass or luminosity, rather than to a systematic variation in mass-dynamical structure. The SLACS sample shows no significant correlation between luminosity and Sérsic index $n$.

10. Expressed as a function of luminosity, the central $V$-band total mass-to-light ratio $\Upsilon_{V, e 2}$ of SLACS lenses scales according to $\log _{10}\left(\Upsilon_{V, e 2} / \Upsilon_{V, \odot}=\right.$ $(0.83 \pm 0.01)+(0.37 \pm 0.06) \log _{10}\left(L_{V} / 10^{11} L_{V, \odot}\right)$.

11. We obtain a nearly model-independent ensemble constraint on the average mass-density profile in the SLACS sample by assuming (in accordance with the FP) that the lenses form a two-parameter family. We scale strong-lensing aperture masses by $M_{\mathrm{dim}}$ and radial mass apertures (Einstein radii) by $R_{e}$, and find a non-dimensional projected massradius relation given by $\log _{10}\left[M(<R) / M_{\text {dim }}\right]=$ $(1.10 \pm 0.09) \log _{10}\left[R / R_{e}\right]+(0.85 \pm 0.03)$. This result is inconsistent with the steeper slope of the projected aperture luminosity profile at a level of significance between $99.9 \%$ and $99.99 \%$, thus falsifying the light-traces-mass hypothesis. If we assume that all projected mass interior to $0.3 R_{e}$ is in the form of stars - a "maximal bulge" assumptionthis result translates into a lower limit on the average projected dark-matter fraction of $f_{\mathrm{DM}}=$ $0.38 \pm 0.07$ inside one effective radius. The threedimensional mass-density profile corresponding to our two-dimensional result is $\rho(r) \propto r^{-1.90 \pm 0.09}$, consistent with the isothermal (flat rotation curve) model.

\section{DISCUSSION AND CONCLUSIONS}

The SLACS lens sample provides a unique resource for the quantitative study of the mass-dynamical structure of massive early-type galaxies. This is due to the addition of strong-lensing aperture masses to a full complement of traditional galaxy observables over a significant range of intrinsic parameter variation. The fact that the SLACS gravitational lens sample defines a fundamental plane similar to that defined by the larger sample of SDSS early-type galaxies suggests that deductions based upon the SLACS lenses can be generalized to early-type galaxies in general. This conclusion is further supported by the FP analysis of Paper II, and by the analysis in $\mathrm{Pa}-$ per $\mathrm{V}$ of the distribution of SLACS lenses in luminosity within their parent samples.

\subsection{The mass-velocity connection}

The lack of correlation between $f \equiv \sigma_{\mathrm{e} 8} / \sigma_{\mathrm{SIE}}$ and either mass or $R_{\mathrm{Ein}} / R_{e}$ in fact contains the essence of our results on the mass independence of galaxy structure and the near isothermal nature of the radial profile, as we now illustrate. Consider the following relation for the mass enclosed within the Einstein radius, which is a consequence of lensing geometry and holds for all mass density models (e.g., Narayan \& Bartelmann 1996):

$$
M\left(R_{\text {Ein }}\right)=M\left(b D_{\mathrm{L}}\right)=\frac{c^{2} b^{2}}{4 G} \frac{D_{\mathrm{L}} D_{\mathrm{S}}}{D_{\mathrm{LS}}} .
$$

Furthermore, take Equation 1 as the definition of the lens-model velocity dispersion parameter $\sigma_{\mathrm{SIE}}$ in terms of the observable angular Einstein radius $b$ (which is in turn related to the physical Einstein radius through $R_{\text {Ein }}=$ $\left.b D_{\mathrm{L}}\right)$. Now consider an idealized case where $R_{\text {Ein }}=R_{e} / 2$ across a range of masses. Equation 9 becomes

$$
\begin{aligned}
M\left(R_{e} / 2\right) & =\pi\left(\frac{c^{2} b}{4 \pi} \frac{D_{\mathrm{S}}}{D_{\mathrm{LS}}}\right)\left(\frac{R_{e} / 2}{G}\right) \\
& =\pi\left(\sigma_{\mathrm{SIE}}^{2}\right)\left(\frac{M_{\mathrm{dim}}}{\sigma_{\mathrm{stars}}^{2}}\right) \\
& =\pi f^{-2} M_{\mathrm{dim}} .
\end{aligned}
$$

Thus the lack of a correlation of $f$ with $M_{\text {dim }}$ implies a linear relationship between $M\left(R_{e} / 2\right)$ and $M_{\mathrm{dim}}$. Similarly, if we now consider $R=R_{\text {Ein }}$ for any fraction of $R_{e}$ and divide Equation 9 by $M_{\mathrm{dim}}$, we obtain

$$
\begin{aligned}
\frac{M(R)}{M_{\mathrm{dim}}} & =\pi\left(\frac{c^{2} b}{4 \pi} \frac{D_{\mathrm{S}}}{D_{\mathrm{LS}}}\right)\left(\frac{R}{G}\right)\left(\frac{G}{\sigma_{\text {stars }}^{2} R_{e} / 2}\right) \\
& =2 \pi \sigma_{\mathrm{SIE}}^{2}\left[R /\left(\sigma_{\mathrm{stars}}^{2} R_{e}\right)\right] \\
& =2 \pi f^{-2}\left(R / R_{e}\right) .
\end{aligned}
$$

Thus a non-isothermal mass profile, in which the enclosed mass does not grow linearly with radius, would appear as a correlation of $f$ with the aperture-radius ratio $R / R_{e}$.

\subsection{The FP-structure connection}

The FP is often contrasted with the so-called "virial expectation", but there is in fact no a priori reason to expect that either the stellar mass-to-light ratio or the central dark-matter fraction should be constant with galaxy mass. Nevertheless, the existence of the FP of elliptical 
galaxies implies a regularity in their formation and evolution history. Our result explains this regularity in terms of a universal mass-dynamical structure which is the end state of massive elliptical evolution regardless of mass, together with a systematic trend in total (luminous plus dark) mass-to-light ratio with galaxy mass. As discussed in $\$ 6$, there is no apparent trend of Sérsic index $n$ with luminosity within the SLACS sample. Despite this fact, the SLACS lenses define a clear FP relationship; the explanation of the SLACS FP must therefore lie with other factors. We reiterate here, though, that the SLACS lens sample is confined to the high-mass end of the elliptical galaxy population. Thus we cannot rule out the importance of the Sérsic index to the FP of lower-mass earlytype galaxies. An important implication of our result is that the "dynamical mass" can be used as a suitable proxy for the true mass inside $1-10 \mathrm{kpc}$, with a conversion factor that is independent of galaxy mass or size. A similar result has been reported by Cappellari et al. (2006) for their dynamical analysis of a mostly lowermass galaxy sample.

In the strictest sense, the fact that we find no massdependent trend in the ratio of dynamical mass to true mass is suggestive of a universal mass-dynamical structure, but not fully conclusive. In principle, a combination of various other dynamical details - perhaps trends in the anisotropy profile of the stellar orbits, perhaps trends in the extent of dynamical relaxationcould conspire to give an unchanging dimensionless structure constant with mass. We also note that the massredshift degeneracy in the SLACS sample could in principle also be masking a mass-dependent trend if significant dynamical evolution occurs with redshift (see, e.g., van der Marel \& van Dokkum 2007a,b), and the more massive lens galaxies evolve to have the same dynamical structure as the less massive lens galaxies at lower redshift.

\subsection{Stellar mass or dark mass?}

Gravitational lensing in both strong and weak forms measures total mass: stars and dark matter together. ${ }^{14}$ A similar statement holds for mass constraints based upon dynamical measurements (which we note here even though our use of stellar dynamics in this paper is limited to empiricism and dimensional analysis): although the dynamical tracers are distributed with the optical luminosity, their orbits are determined by the potential of the total (luminous plus dark) matter distribution. The results presented here strongly suggest that the total mass-density structure of elliptical galaxies is universali.e., not a function of the other observables - at least over the range of galaxy masses covered by the SLACS sample. But what is the breakdown of this mass into stellar and dark-matter components? Fundamentally, this question cannot be answered without the imposition of prior conditions on either the form of the dark-matter density profile or the mass-to-light ratio of the stellar component.

\footnotetext{
14 Gravitational microlensing, in contrast, can distinguish between mass distributed among point-like stellar-mass objects and mass distributed in a smooth, continuous component. See, e.g., Deguchi \& Watson (1987, 1988); Webster et al. (1991); Seitz, Wambsganss, \& Schneider (1994); Witt, Mao, \& Schechter (1995); Lewis \& Irwin (1995, 1996); Schechter \& Wambsganss (2002)
}

To express the ambiguity mathematically, for any stellar and dark-matter density profiles $\rho_{\star}$ and $\rho_{\text {DM }}$ that satisfy all observational lensing and dynamical constraints, we may transform according to

$$
\begin{gathered}
\rho_{\star} \longrightarrow \rho_{\star}^{\prime}=\beta \rho_{\star} \\
\rho_{\mathrm{DM}} \longrightarrow \rho_{\mathrm{DM}}^{\prime}=\rho_{\mathrm{DM}}+(1-\beta) \rho_{\star},
\end{gathered}
$$

subject only to the requirement that $\rho_{\star}^{\prime}>0$ and $\rho_{\mathrm{DM}}^{\prime}>0$ everywhere, without altering either the observable quantities or the internal dynamical consistency of the system. Previous papers in the SLACS series (Paper II, Paper III, Paper IV) have approached this problem from both the dark-matter profile and stellar-population angles. With forthcoming multi-band photometric data, we will be able to estimate stellar masses through more detailed stellar-population modeling, which we may in turn relate to lensing and dynamical masses to establish overall trends within the population. (see, e.g., Gallazzi et al. 2006; Bundy et al. 2007; Grillo et al. 2008).

Qualitatively, our result is in good agreement with the recent theoretical work of Bovlan-Kolchin, Ma, \& Quataert (2005) and Robertson et al. (2006). Based on the analysis of numerical simulations, these authors argue that the tilt of the FP is the result of a systematic trend in the central dark-matter fraction that is established and preserved though the assembly of successively more massive spheroids via mergers. Robertson et al. (2006) argue further that this trend is originally established through the importance of dissipative gas processes in disk galaxy formation and early merging. Similar conclusions were reached observationally by Padmanabhan et al. (2004) with the aid of stellar-population modeling and theoretically-motivated dark-matter halo models, and by Cappellari et al. (2006) through the combination of spatially resolved dynamical modeling with stellar population modeling.

\subsection{Final thoughts}

In conclusion, our results offer the following physical picture for the tilt and tightness of the FP for massive early-type galaxies (see also the discussion of Paper III). The luminosity profiles are, on average, well described by a de Vaucouleurs model luminosity profile. This profile is embedded in an on-average scale-free isothermal $\left(\rho \propto r^{-2}\right)$ total mass density profile, with the distribution of the dark matter dictated by a bulge-halo "conspiracy" to establish the isothermal total profile. The conspiracy is not far-fetched if the isothermal profile is in fact a dynamical attractor for the evolution of centrally condensed self-gravitating particle systems, since stars and cold dark matter together constitute a single collisionless component from the point of view of gravitational dynamics (Loeb \& Peebles 2003). The galaxies thus form a two-parameter family, indexed by the effective radius of the stellar distribution (the only observable scale length) and the central velocity dispersion of the system (the only parameter of the mass model). Luminosity (and hence mass-to-light ratio) then varies systematically with the scale size of the stellar distribution in such a way as to give a gradual increase in the total mass-to-light ratio with increasing total mass within the stellar effective radius. 
Observationally, this picture remains ambiguous as to the systematic breakdown of mass into stars and dark matter along the FP, a deficiency that can be partly addressed through analysis of forthcoming multi-band $H S T$ imaging (ACS, WFPC2 and NICMOS) of the SLACS lens sample, which will give better insight into the role of stellar-population effects in establishing the scaling relations examined here. In addition, spatially resolved kinematic data are being obtained for the SLACS lenses (Czoske et al. 2008) to gain the tightest possible constraints on the possible luminous/dark decompositions (e.g., Barnabè \& Koopmans 2007). This ongoing analysis will lead to a clearer and more robust picture of the empirical physical relations described in this work.

ASB, TT, LVEK, RG, and LAM acknowledge the support and hospitality of the Kavli Institute for Theoretical Physics at UCSB, where the early stages of this work were completed. ASB thanks G. Dobler, G. Novak, and S. Faber for valuable discussion related to this work. TT acknowledges support from the NSF through CAREER award NSF-0642621 and from the Sloan Foundation through a Sloan Research Fellowship. He is also supported by a Packard fellowship. LVEK is supported in part through an NWO-VIDI program subsidy (project number 639.042.505). He also acknowledges the continuing support by the European Community's Sixth Framework Marie Curie Research Training Network Programme, Contract No. MRTN-CT-2004-505183 "ANGLES"). The work of LAM was carried out at Jet Propulsion Laboratory, California Institute of Technology under a contract with NASA.

Support for HST programs \#10174, \#10494, \#10587,
\#10798, and \#10886 was provided by NASA through a grant from the Space Telescope Science Institute, which is operated by the Association of Universities for Research in Astronomy, Inc., under NASA contract NAS 5-26555. Please see HST data acknowledgment on title page.

This work has made extensive use of the Sloan Digital Sky Survey database. Funding for the SDSS and SDSS-II has been provided by the Alfred P. Sloan Foundation, the Participating Institutions, the National Science Foundation, the U.S. Department of Energy, the National Aeronautics and Space Administration, the Japanese Monbukagakusho, the Max Planck Society, and the Higher Education Funding Council for England. The SDSS Web Site is http: //www.sdss.org/.

The SDSS is managed by the Astrophysical Research Consortium for the Participating Institutions. The Participating Institutions are the American Museum of Natural History, Astrophysical Institute Potsdam, University of Basel, University of Cambridge, Case Western Reserve University, University of Chicago, Drexel University, Fermilab, the Institute for Advanced Study, the Japan Participation Group, Johns Hopkins University, the Joint Institute for Nuclear Astrophysics, the Kavli Institute for Particle Astrophysics and Cosmology, the Korean Scientist Group, the Chinese Academy of Sciences (LAMOST), Los Alamos National Laboratory, the Max-Planck-Institute for Astronomy (MPIA), the MaxPlanck-Institute for Astrophysics (MPA), New Mexico State University, Ohio State University, University of Pittsburgh, University of Portsmouth, Princeton University, the United States Naval Observatory, and the University of Washington.

\section{REFERENCES}

Adelman-McCarthy, J. K., et al. 2007, ApJS, in press (ArXiv 0707.3413)

Auger, M. W. 2007, MNRAS, L127+

Barnabè, M., \& Koopmans, L. V. E. 2007, ApJ, 666, 726

Belokurov, V., et al. 2007, ApJ, 671, L9

Bender, R., Burstein, D., \& Faber, S. M. 1992, ApJ, 399, 462

Bender, R., Saglia, R. P., \& Gerhard, O. E. 1994, MNRAS, 269, 785

Bernardi, M., et al. 2003, AJ, 125, 1866

Bertin, G., Ciotti, L., \& Del Principe, M. 2002, A\&A, 386, 149

Bertin, G., Saglia, R. P., \& Stiavelli, M. 1992, ApJ, 384, 423

Bertin, G., et al. 1994, A\&A, 292, 381

Bertola, F., \& Capaccioli, M. 1975, ApJ, 200, 439

Binney, J. 1976, MNRAS, 177, 19

-. 1978, MNRAS, 183, 501

Blackburne, J. A., Wisotzki, L., \& Schechter, P. L. 2008, AJ, 135, 374

Bolton, A. S., Burles, S., Koopmans, L. V. E., Treu, T., \& Moustakas, L. A. 2006, ApJ, 638, 703 (Paper I)

Bolton, A. S., Burles, S., Treu, T., Koopmans, L. V. E., \& Moustakas, L. A. 2007, ApJ, 665, L105 (B07)

Bolton, A. S., et al. 2008, ApJ, in press (Paper V)

Boylan-Kolchin, M., Ma, C.-P., \& Quataert, E. 2005, MNRAS, 362, 184

Brewer, B. J., \& Lewis, G. F. 2006, ApJ, 651, 8

Browne, I. W. A., et al. 2003, MNRAS, 341, 13

Bundy, K., Treu, T., \& Ellis, R. S. 2007, ApJ, 665, L5

Cabanac, R. A., et al. 2007, A\&A, 461, 813

Caon, N., Capaccioli, M., \& D'Onofrio, M. 1993, MNRAS, 265, 1013

Cappellari, M., et al. 2006, MNRAS, 366, 1126

Ciotti, L., Lanzoni, B., \& Renzini, A. 1996, MNRAS, 282, 1
Cohn, J. D., Kochanek, C. S., McLeod, B. A., \& Keeton, C. R. 2001, ApJ, 554, 1216

Cowie, L. L., Songaila, A., Hu, E. M., \& Cohen, J. G. 1996, AJ, 112,839

Czoske, O., Barnabè, M., Koopmans, L. V. E., Treu, T., \&

Bolton, A. S. 2008, MNRAS, 384, 987

Deguchi, S., \& Watson, W. D. 1987, Physical Review Letters, 59, 2814

-. 1988, ApJ, 335, 67

di Serego Alighieri, S., et al. 2005, A\&A, 442, 125

Djorgovski, S., \& Davis, M. 1987, ApJ, 313, 59

D'Onofrio, M., Capaccioli, M., \& Caon, N. 1994, MNRAS, 271, 523

Dressler, A., Lynden-Bell, D., Burstein, D., Davies, R. L., Faber, S. M., Terlevich, R., \& Wegner, G. 1987, ApJ, 313, 42

Dye, S., Smail, I., Swinbank, A. M., Ebeling, H., \& Edge, A. C. 2007, MNRAS, 379, 308

Dye, S., \& Warren, S. J. 2005, ApJ, 623, 31

Efron, B. 1979, Ann. Stat., 7, 1

Faber, S. M., Dressler, A., Davies, R. L., Burstein, D., \& Lynden-Bell, D. 1987, in Nearly Normal Galaxies. From the

Planck Time to the Present, ed. S. M. Faber, 175-183

Faure, C., et al. 2008, ApJ, in press (arXiv:0802.2174)

Ferrarese, L., et al. 2006, ApJS, 164, 334

Gallazzi, A., Charlot, S., Brinchmann, J., \& White, S. D. M. 2006, MNRAS, 370, 1106

Gavazzi, R., et al. 2007, ApJ, 667, 176 (Paper IV)

—. 2008, ApJ, 677, 1046 (Paper VI)

Gerhard, O., Kronawitter, A., Saglia, R. P., \& Bender, R. 2001, AJ, 121, 1936

Gerhard, O. E. 1993, MNRAS, 265, 213

Graham, A., \& Colless, M. 1997, MNRAS, 287, 221

Graham, A. W., \& Guzmán, R. 2003, AJ, 125, 2936 
Gregg, M. D., et al. 2000, AJ, 119, 2535

Grillo, C., Gobat, R., Rosati, P., \& Lombardi, M. 2008, A\&A, 477, L25

Guzmán, R., Lucey, J. R., \& Bower, R. G. 1993, MNRAS, 265, 731

Hjorth, J., \& Madsen, J. 1995, ApJ, 445, 55

Illingworth, G. 1977, ApJ, 218, L43

Inada, N., et al. 2007, ArXiv e-prints, 708

Jørgensen, I., Franx, M., \& Kjærgaard, P. 1995, MNRAS, 276, 1341

-. 1996, MNRAS, 280, 167

Kassiola, A., \& Kovner, I. 1993, ApJ, 417, 450

Keeton, C. R., \& Kochanek, C. S. 1998, ApJ, 495, 157

Kelson, D. D., van Dokkum, P. G., Franx, M., Illingworth, G. D., \& Fabricant, D. 1997, ApJ, 478, L13+

Kochanek, C. S. 1991, ApJ, 373, 354

-. 1994, ApJ, 436, 56

-. 2002, ApJ, 578, 25

Kochanek, C. S., Morgan, N. D., Falco, E. E., McLeod, B. A.,

Winn, J. N., Dembicky, J., \& Ketzeback, B. 2006, ApJ, 640, 47

Kochanek, C. S., et al. 2000, ApJ, 543, 131

Koopmans, L. V. E., \& Treu, T. 2002, ApJ, 568, L5

—. 2003, ApJ, 583, 606

Koopmans, L. V. E., Treu, T., Bolton, A. S., Burles, S., \& Moustakas, L. A. 2006, ApJ, 649, 599 (Paper III)

Kormann, R., Schneider, P., \& Bartelmann, M. 1994, A\&A, 284, 285

Kronawitter, A., Saglia, R. P., Gerhard, O., \& Bender, R. 2000, A\&AS, 144, 53

Kubo, J. M., \& Dell'Antonio, I. P. 2008, MNRAS, in press

Kuijken, K., \& Merrifield, M. R. 1993, MNRAS, 264, 712

Lewis, G. F., \& Irwin, M. J. 1995, MNRAS, 276, 103

-. 1996, MNRAS, 283, 225

Loeb, A., \& Peebles, P. J. E. 2003, ApJ, 589, 29

Maoz, D., et al. 1993, ApJ, 409, 28

Merritt, D., \& Saha, P. 1993, ApJ, 409, 75

Morgan, N. D., Gregg, M. D., Wisotzki, L., Becker, R., Maza, J., Schechter, P. L., \& White, R. L. 2003, AJ, 126, 696

Moustakas, L. A., et al. 2007, ApJ, 660, L31

Muñoz, J. A., Kochanek, C. S., \& Keeton, C. R. 2001, ApJ, 558, 657

Myers, S. T., et al. 2003, MNRAS, 341, 1

Narayan, R., \& Bartelmann, M. 1996, astro-ph/9606001

Oguri, M., et al. 2006, AJ, 132, 999

-. 2007, ArXiv e-prints, 708

Padmanabhan, N., et al. 2004, New Astronomy, 9, 329

Pahre, M. A., de Carvalho, R. R., \& Djorgovski, S. G. 1998a, AJ, 116,1606

Pahre, M. A., Djorgovski, S. G., \& de Carvalho, R. R. 1995, ApJ, 453, L17+

-. 1998b, AJ, 116, 1591

Ratnatunga, K. U., Griffiths, R. E., \& Ostrander, E. J. 1999, AJ, 117,2010

Renzini, A., \& Ciotti, L. 1993, ApJ, 416, L49+

Rix, H.-W., de Zeeuw, P. T., Cretton, N., van der Marel, R. P., \& Carollo, C. M. 1997, ApJ, 488, 702

Rix, H.-W., \& White, S. D. M. 1992, MNRAS, 254, 389

Robertson, B., Cox, T. J., Hernquist, L., Franx, M., Hopkins, P. F., Martini, P., \& Springel, V. 2006, ApJ, 641, 21

Romanowsky, A. J., \& Kochanek, C. S. 2001, ApJ, 553, 722

Rusin, D., \& Kochanek, C. S. 2005, ApJ, 623, 666

Rusin, D., Kochanek, C. S., \& Keeton, C. R. 2003, ApJ, 595, 29

Saglia, R. P., Bertin, G., \& Stiavelli, M. 1992, ApJ, 384, 433

Saglia, R. P., Kronawitter, A., Gerhard, O., \& Bender, R. 2000, AJ, 119, 153
Saglia, R. P., et al. 1993, ApJ, 403, 567

Schechter, P. L., \& Wambsganss, J. 2002, ApJ, 580, 685

Schlegel, D. J., Finkbeiner, D. P., \& Davis, M. 1998, ApJ, 500, 525

Schneider, P., Kochanek, C. S., \& Wambsganss, J. 2006, in Saas-Fee Advanced Course 33: Gravitational Lensing: Strong, Weak and Micro (Part 2 available as astro-ph/0407232), ed.

G. Meylan, P. Jetzer, \& P. North (Berlin: Springer)

Scodeggio, M., Gavazzi, G., Belsole, E., Pierini, D., \& Boselli, A. 1998, MNRAS, 301, 1001

Seitz, C., Wambsganss, J., \& Schneider, P. 1994, A\&A, 288, 19

Treu, T., Ellis, R. S., Liao, T. X., \& van Dokkum, P. G. 2005a, ApJ, 622, L5

Treu, T., Koopmans, L. V., Bolton, A. S., Burles, S., \& Moustakas, L. A. 2006, ApJ, 640, 662 (Paper II)

Treu, T., \& Koopmans, L. V. E. 2002, ApJ, 575, 87

-. 2003, MNRAS, 343, L29

-. 2004, ApJ, 611, 739

Treu, T., Stiavelli, M., Bertin, G., Casertano, S., \& Møller, P. 2001, MNRAS, 326, 237

Treu, T., Stiavelli, M., Casertano, S., Møller, P., \& Bertin, G. 2002, ApJ, 564, L13

Treu, T., et al. 2005b, ApJ, 633, 174

Trujillo, I., Burkert, A., \& Bell, E. F. 2004, ApJ, 600, L39

van de Ven, G., van Dokkum, P. G., \& Franx, M. 2003, MNRAS, 344,924

van der Marel, R. P. 1991, MNRAS, 253, 710

van der Marel, R. P., \& Franx, M. 1993, ApJ, 407, 525

van der Marel, R. P., \& van Dokkum, P. G. 2007a, ApJ, 668, 738

-. 2007b, ApJ, 668, 756

van der Wel, A., Franx, M., van Dokkum, P. G., Huang, J., Rix,

H.-W., \& Illingworth, G. D. 2006, ApJ, 636, L21

van der Wel, A., Franx, M., van Dokkum, P. G., Rix, H.-W.,

Illingworth, G. D., \& Rosati, P. 2005, ApJ, 631, 145

van Dokkum, P. G., \& Franx, M. 1996, MNRAS, 281, 985

van Dokkum, P. G., \& Stanford, S. A. 2003, ApJ, 585, 78

Wayth, R. B., Warren, S. J., Lewis, G. F., \& Hewett, P. C. 2005, MNRAS, 360, 1333

Webster, R. L., Ferguson, A. M. N., Corrigan, R. T., \& Irwin, M. J. 1991, AJ, 102, 1939

Willis, J. P., Hewett, P. C., \& Warren, S. J. 2005, MNRAS, 363, 1369

Willis, J. P., Hewett, P. C., Warren, S. J., Dye, S., \& Maddox, N. 2006, MNRAS, 369, 1521

Winn, J. N., Lovell, J. E. J., Chen, H.-W., Fletcher, A. B.,

Hewitt, J. N., Patnaik, A. R., \& Schechter, P. L. 2002a, ApJ, 564,143

Winn, J. N., et al. 2000, AJ, 120, 2868

—. 2001, AJ, 121, 1223

-. 2002b, AJ, 123, 10

Wisotzki, L., Christlieb, N., Liu, M. C., Maza, J., Morgan, N. D., \& Schechter, P. L. 1999, A\&A, 348, L41

Wisotzki, L., Koehler, T., Kayser, R., \& Reimers, D. 1993, A\&A, 278, L15

Wisotzki, L., Koehler, T., Lopez, S., \& Reimers, D. 1996, A\&A, $315, \mathrm{~L} 405+$

Wisotzki, L., Schechter, P. L., Bradt, H. V., Heinmüller, J., \& Reimers, D. 2002, A\&A, 395, 17

Wisotzki, L., Schechter, P. L., Chen, H.-W., Richstone, D., Jahnke, K., Sánchez, S. F., \& Reimers, D. 2004, A\&A, 419, L31

Witt, H. J., Mao, S., \& Schechter, P. L. 1995, ApJ, 443, 18

York, D. G., et al. 2000, AJ, 120, 1579 